\title{
Funções auxiliares nos teoremas clássicos do valor médio
}

\author{
German Lozada-Cruz (i)
}

\begin{abstract}
Resumo
Neste trabalho mostramos algumas variantes dos teorema clássicos do valor médio. As ferramentas principais para mostrarmos estes resultados são funções auxiliares elementares.

Palavras-chave: Teorema do Valor Médio de Lagrange; Teorema do Valor Médio de Cauchy; Teorema do Valor Médio para Integrais.

\section{Abstract}

In this work, some variants of classical mean value theorems are proved. The main tools to prove these results are some elementary auxiliary functions.
\end{abstract}

Keyword: Lagrange's mean value theorem; Cauchy's mean value theorem; Integral mean value theorem.

\section{Introdução}

Sabemos que o primeiro contato de nossos alunos dos cursos de graduação em Matemática ou de Engenharia com os teoremas do tipo valor médio é em um curso de Cálculo Diferencial e Integral ([11] e [17] por exemplo) ou em um primeiro curso de Análise Real ([12]).

O primeiro teorema do valor médio é o conhecido Teorema do Valor Médio de Lagrange, o qual relaciona a taxa média da variação de uma função nos extremos de um intervalo com o valor da derivada da função em um ponto do mesmo intervalo.

O segundo teorema de valor médio é o Teorema do Valor Médio de Cauchy, o qual é uma generalização do Teorema do Valor Médio de Lagrange, que estabelece uma relação entre as derivadas de duas funções e a variação dessas funções em um intervalo finito.

O terceiro teorema de valor médio é o Teorema do Valor Médio para Integrais ou simplesmente Teorema do Valor Médio Integral, que relaciona a área da região sob o gráfico de uma função real $\mathrm{f}$ definida num intervalo fechado [a,b] com a área de um retângulo de base b-a e altura f(c) para algum c no intervalo [a, b].

Nas demonstrações desses teoremas (veja por exemplo [3, Teorema 2.3] e [12, Teorema 4.12]) algumas funções auxiliares são usadas e então aplica-se diretamente o Teorema de Rolle: Seja $\mathrm{f}:[\mathrm{a}, \mathrm{b}] \rightarrow \mathbb{R}$ contínua em $[\mathrm{a}, \mathrm{b}]$ e diferenciável em $(\mathrm{a}, \mathrm{b})$. Se $\mathrm{f}(\mathrm{a})=\mathrm{f}(\mathrm{b})$, então, existe $\mathrm{c} \in(\mathrm{a}, \mathrm{b})$ tal que $\mathrm{f}^{\prime}(\mathrm{c})=0$. Alguns autores chamam isso de "ideia feliz" ([7, p.24]).

O objetivo principal deste trabalho é usar funções auxiliares elementares para demonstrar algumas variações tanto dos Teoremas do Valor Médio de Lagrange, de Cauchy e Integral. 


\section{Funções auxiliares para o Teorema do Valor Médio de Lagrange}

Nesta seção vamos usar algumas funções auxiliares elementares para demonstrar algumas variações do Teorema do Valor Médio de Lagrange.

Teorema 1 (Teorema de Lagrange). Se $\mathrm{f}:[\mathrm{a}, \mathrm{b}] \rightarrow \mathbb{R}$ é uma função contínua em $[\mathrm{a}, \mathrm{b}]$ e derivável em $(\mathrm{a}, \mathrm{b})$, então existe $\eta \in(\mathrm{a}, \mathrm{b})$ tal que

$$
\mathrm{f}(\eta)-\mathrm{f}(\mathrm{a})=\mathrm{f}^{\prime}(\eta)(\eta-\mathrm{a}) .
$$

Geometricamente, o Teorema do Valor Médio de Lagrange diz-nos que existe um ponto $\eta$ dentro do intervalo $(\mathrm{a}, \mathrm{b})$, onde a reta tangente ao gráfico de $\mathrm{f}$ é paralela à reta secante que passa pelos pontos $A=(a, f(a))$ e $B=(b, f(b))$, ou seja $f^{\prime}(\eta)=\frac{f(b)-f(a)}{b-a}$.

Se $f(t)$ representa a posição de um corpo que se move ao longo de uma reta, dependendo do tempo $\mathrm{t}$, então o quociente $\frac{\mathrm{f}(\mathrm{b})-\mathrm{f}(\mathrm{a})}{\mathrm{b}-\mathrm{a}}$ é a velocidade média do corpo no período de tempo b-a. Como $\mathrm{f}^{\prime}(\mathrm{t})$ é a velocidade instantânea, fisicamente o Teorema do Valor Médio de Lagrange diz que existe um instante de tempo $\eta$, para o qual a velocidade instantânea é igual à velocidade média.

A demonstração do Teorema de Lagrange (veja [12, Teorema 4.12]) consiste em aplicar o Teorema de Rolle à função auxiliar

$$
\phi(x)=f(x)-f(a)-\frac{f(b)-f(a)}{b-a}(x-a)
$$

Neste ponto, cabe fazer a seguinte pergunta: Qual é a origem da função auxiliar $\phi$ usada na demonstração do Teorema do Valor Médio de Lagrange?

A explicação mais simples da escolha de $\phi$ provavelmente seja a dada por R.C. Yates ([23]). A função $\phi$ é a diferença da ordenada do ponto $\mathrm{P}=(\mathrm{x}, \mathrm{f}(\mathrm{x}))$ no gráfico de $\mathrm{f}$ e da ordenada do ponto $\mathrm{Q}=(\mathrm{x}, \mathrm{h}(\mathrm{x}))$ na reta secante ao gráfico de $\mathrm{f}$. Como as funções $\mathrm{f}$ e $\mathrm{h}$ se encontram nos pontos $(\mathrm{a}, \mathrm{f}(\mathrm{a}))$ e $(\mathrm{b}, \mathrm{f}(\mathrm{b}))$ temos $\phi(\mathrm{a})=0=\phi(\mathrm{b})$. Assim, temos uma função para a qual podemos aplicar o Teorema de Rolle; consequentemente, obtemos o Teorema do Valor Médio de Lagrange.

Existem outras maneiras de obter $\phi$ (veja [10], [15] por exemplo). M.R. Spiegel em [16] observou que podemos encontrar $\phi$ da seguinte forma: vamos procurar uma aproximação linear de f, $\alpha+\beta \mathrm{x}$, no intervalo [a,b], onde $\alpha$ e $\beta$ são constantes a serem determinadas. Agora consideremos a diferença, $\phi(\mathrm{x})$, entre $\mathrm{f}(\mathrm{x})$ e sua aproximação linear $\alpha+\beta \mathrm{x}$, i.e.,

$$
\phi(\mathrm{x})=\mathrm{f}(\mathrm{x})-(\alpha+\beta \mathrm{x})
$$

onde $\alpha$ e $\beta$ são números reais a serem determinados satisfazendo a condição $\phi(\mathrm{a})=\phi(\mathrm{b})$. Dessa última condição obtemos $\beta=\frac{\mathrm{f}(\mathrm{b})-\mathrm{f}(\mathrm{a})}{\mathrm{b}-\mathrm{a}}$. Para acharmos $\alpha$, fazemos $\phi(\mathrm{a})=0$, assim obtemos $\alpha=\mathrm{f}(\mathrm{a})-\frac{\mathrm{f}(\mathrm{b})-\mathrm{f}(\mathrm{a})}{\mathrm{b}-\mathrm{a}} \mathrm{a}$. Logo,

$$
\phi(x)=f(x)-f(a)-\frac{f(b)-f(a)}{b-a}(x-a)
$$

Se fizermos uma ligeira modificação da aproximação linear de f da forma

$$
\phi(\mathrm{x})=\mathrm{f}(\mathrm{x})+\lambda \mathrm{x}
$$


onde $\lambda \in \mathbb{R}$ tal que a condição $\phi(\mathrm{a})=\phi(\mathrm{b})$ seja satisfeita, obtemos a seguinte função auxiliar

$$
\phi(x)=f(x)-\frac{f(b)-f(a)}{b-a} x .
$$

J.Tong em [18] introduziu a função auxiliar da forma

$$
H(x)=f(x)-\frac{f(b)-f(a)}{b-a}\left(x-\frac{a+b}{2}\right),
$$

a qual satisfaz a condição $H(a)=\frac{f(a)+f(a)}{2}=H(b)$.

Se mudarmos ligeiramente a função auxiliar H dada em (5) pela função auxiliar

$$
H(x)=f(x)-\frac{f(a)+f(b)}{2}-\frac{f(b)-f(a)}{b-a}\left(x-\frac{a+b}{2}\right)
$$

Facilmente vemos que $\mathrm{H}(\mathrm{a})=0=\mathrm{H}(\mathrm{b})$.

J.Tong em [19, Teorema 1] exibe uma família de funções auxiliares que envolvem dois parâmetros $\alpha$ e $\beta$, os quais satisfazem o Teorema de Rolle. Consequentemente, obtemos uma família de Teoremas de Valor Médio. Em particular, quando $\alpha=\beta$, obtém-se o Teorema do Valor Médio de Lagrange. Isso leva a concluir que existem uma infinidade de funções auxiliares.

Em 1958 T.M. Flett em [2] enunciou e demonstrou uma variação do Teorema de Lagrange.

Teorema 2 (Teorema de Flett [2]). Se $\mathrm{f}:[\mathrm{a}, \mathrm{b}] \rightarrow \mathbb{R}$ é uma função derivável em $[\mathrm{a}, \mathrm{b}]$ e $\mathrm{f}^{\prime}(\mathrm{a})=\mathrm{f}^{\prime}(\mathrm{b})$, então, existe $\eta \in(\mathrm{a}, \mathrm{b})$ tal que

$$
\mathrm{f}(\eta)-\mathrm{f}(\mathrm{a})=\mathrm{f}^{\prime}(\eta)(\eta-\mathrm{a})
$$

Geometricamente, o Teorema de Flett diz que se uma curva (t,f(t)) é suave no intervalo [a,b] e as retas tangentes nos extremos $(\mathrm{a}, \mathrm{f}(\mathrm{a}))$ e $(\mathrm{b}, \mathrm{f}(\mathrm{b}))$ são paralelas, então, existe um ponto $\eta \in(\mathrm{a}, \mathrm{b})$ de modo que a reta tangente ao gráfico de $\mathrm{f}$ que passa por $(\eta, \mathrm{f}(\eta))$ também passa por $(\mathrm{a}, \mathrm{f}(\mathrm{a}))$.

Fisicamente, o Teorema de Flett diz que, se as velocidades inicial e final de uma partícula com trajetória $(\mathrm{t}, \mathrm{f}(\mathrm{t}))$ suave no intervalo de tempo $[\mathrm{a}, \mathrm{b}]$ forem iguais, então, existe um momento $\eta \in(\mathrm{a}, \mathrm{b})$ tal que a velocidade instantânea da partícula nesse instante, é exatamente a velocidade média do percurso até o instante $\eta$.

Em 1977, R.E. Myers [9] demonstrou uma variação do Teorema de Flett.

Teorema 3 (Teorema de Myers [9]). Se $\mathrm{f}:[\mathrm{a}, \mathrm{b}] \rightarrow \mathbb{R}$ é uma função derivável em $[\mathrm{a}, \mathrm{b}]$ e $\mathrm{f}^{\prime}(\mathrm{a})=$ $\mathrm{f}^{\prime}(\mathrm{b})$, então, existe $\eta \in(\mathrm{a}, \mathrm{b})$ tal que

$$
\mathrm{f}(\mathrm{b})-\mathrm{f}(\eta)=\mathrm{f}^{\prime}(\eta)(\mathrm{b}-\eta) .
$$

Em 1998, P.K. Sahoo e T. Riedel ([13, Teorema 5.2]) deram uma variação do Teorema de Flett (Teorema 2) onde eles removeram a condição de fronteira na derivada de f, i.e., $\mathrm{f}^{\prime}(\mathrm{a})=\mathrm{f}^{\prime}(\mathrm{b})$.

Teorema 4 (Teorema de Sahoo-Riedel [13]). Se f : [a,b] $\rightarrow \mathbb{R}$ é uma função derivável em [a,b], 
então, existe $\eta \in(\mathrm{a}, \mathrm{b})$ tal que

$$
\mathrm{f}(\eta)-\mathrm{f}(\mathrm{a})=\mathrm{f}^{\prime}(\eta)(\eta-\mathrm{a})-\frac{1}{2} \frac{\mathrm{f}^{\prime}(\mathrm{b})-\mathrm{f}^{\prime}(\mathrm{a})}{\mathrm{b}-\mathrm{a}}(\eta-\mathrm{a})^{2} .
$$

Demonstração. Aplicar o Teorema de Flett à função auxiliar $\psi:[\mathrm{a}, \mathrm{b}] \rightarrow \mathbb{R}$ dada por

$$
\psi(x)=f(x)-\frac{1}{2} \frac{f^{\prime}(b)-f^{\prime}(a)}{b-a}(x-a)^{2} .
$$

Observação 1. A função auxiliar $\psi$ usada no Teorema de Sahoo-Riedel é obtida considerando a diferença de f com uma aproximação quadrática de f,

$$
\alpha+\beta(\mathrm{x}-\mathrm{a})+\gamma(\mathrm{x}-\mathrm{a})^{2}
$$

numa vizinhança de a, i.e., $\psi(\mathrm{x})=\mathrm{f}(\mathrm{x})-\left[\alpha+\beta(\mathrm{x}-\mathrm{a})+\gamma(\mathrm{x}-\mathrm{a})^{2}\right]$ e impondo a condição de fronteira na derivada de $\psi, \psi^{\prime}(\mathrm{a})=\psi^{\prime}(\mathrm{b})$. Assim,

$$
\begin{aligned}
\psi^{\prime}(\mathrm{a})=\psi^{\prime}(\mathrm{b}) & \Leftrightarrow \mathrm{f}^{\prime}(\mathrm{a})-\beta=\mathrm{f}^{\prime}(\mathrm{b})-\beta-2 \gamma(\mathrm{b}-\mathrm{a}) \\
& \Leftrightarrow \gamma=\frac{1}{2} \frac{\mathrm{f}^{\prime}(\mathrm{b})-\mathrm{f}^{\prime}(\mathrm{a})}{\mathrm{b}-\mathrm{a}} .
\end{aligned}
$$

As constantes $\alpha$ e $\beta$ são arbitrárias e por conveniência podemos tomar $\alpha=0=\beta$.

Da Observação 1 podemos considerar uma função auxiliar adequada para mostrar uma variação do Teorema de Sahoo-Riedel.

Teorema 5 ([4]). Se $\mathrm{f}:[\mathrm{a}, \mathrm{b}] \rightarrow \mathbb{R}$ é uma função diferenciável em $[\mathrm{a}, \mathrm{b}]$, então, existe $\eta \in(\mathrm{a}, \mathrm{b})$ tal que

$$
\mathrm{f}(\eta)-\mathrm{f}(\mathrm{a})=\mathrm{f}^{\prime}(\eta)(\eta-\mathrm{a})-\frac{(\mathrm{n}-1)}{\mathrm{n}} \frac{\mathrm{f}^{\prime}(\mathrm{b})-\mathrm{f}^{\prime}(\mathrm{a})}{(\mathrm{b}-\mathrm{a})^{\mathrm{n}-1}}(\eta-\mathrm{a})^{\mathrm{n}}, \quad \mathrm{n} \in \mathbb{N}
$$

Demonstração. Seja $n \in \mathbb{N}$ e considere a função auxiliar $\psi:[a, b] \rightarrow \mathbb{R}$ dada por

$$
\psi(x)=f(x)+\lambda(x-a)^{n},
$$

onde $\lambda \in \mathbb{R}$ que vamos escolher $\lambda$ tal que a condição $\psi^{\prime}(\mathrm{a})=\psi^{\prime}(\mathrm{b})$ seja satisfeita.

Facilmente vemos que $\psi$ é derivável em $[\mathrm{a}, \mathrm{b}]$ e $\psi^{\prime}(\mathrm{x})=\mathrm{f}^{\prime}(\mathrm{x})+\mathrm{n} \lambda(\mathrm{x}-\mathrm{a})^{\mathrm{n}-1}$. Então,

$$
\begin{aligned}
\psi^{\prime}(\mathrm{a})=\psi^{\prime}(\mathrm{b}) & \Leftrightarrow \mathrm{f}^{\prime}(\mathrm{a})=\mathrm{f}^{\prime}(\mathrm{b})+\mathrm{n} \lambda(\mathrm{b}-\mathrm{a})^{\mathrm{n}-1} \\
& \Leftrightarrow \lambda=-\frac{1}{\mathrm{n}} \frac{\mathrm{f}^{\prime}(\mathrm{b})-\mathrm{f}^{\prime}(\mathrm{a})}{(\mathrm{b}-\mathrm{a})^{\mathrm{n}-1}}
\end{aligned}
$$

Assim, temos a função auxiliar

$$
\psi(x)=f(x)-\frac{1}{n} \frac{f^{\prime}(b)-f^{\prime}(a)}{(b-a)^{n-1}}(x-a)^{n}
$$


, a qual satisfaz as hipóteses do Teorema de Flett (Theorem 2). Então, existe $\eta \in(\mathrm{a}, \mathrm{b})$ tal que

$$
\psi(\eta)-\psi(\mathrm{a})=\psi^{\prime}(\eta)(\eta-\mathrm{a})
$$

Dessa igualdade segue (11).

D.çakmak e A.Tiryaki ([1, Teorema 2.1]) mostrou uma ligeira variação do Teorema de Sahoo-Riedel (Teorema 4) e esse se reduz ao Teorema de Myers (Teorema 3) quando $\mathrm{f}^{\prime}(\mathrm{a})=\mathrm{f}^{\prime}(\mathrm{b})$.

Teorema 6 (Teorema de Çakmak-Tiryaki [1]). Seja $\mathrm{f}:[\mathrm{a}, \mathrm{b}] \rightarrow \mathbb{R}$ uma função diferenciável em $[\mathrm{a}, \mathrm{b}]$, então, existe $\eta \in(\mathrm{a}, \mathrm{b})$ tal que

$$
\mathrm{f}(\mathrm{b})-\mathrm{f}(\eta)=\mathrm{f}^{\prime}(\eta)(\mathrm{b}-\eta)+\frac{1}{2} \frac{\mathrm{f}^{\prime}(\mathrm{b})-\mathrm{f}^{\prime}(\mathrm{a})}{\mathrm{b}-\mathrm{a}}(\mathrm{b}-\eta)^{2} .
$$

Demonstração. Aplicar o Teorema de Myers à função auxiliar $\mathrm{h}:[\mathrm{a}, \mathrm{b}] \rightarrow \mathbb{R}$ definida por

$$
h(x)=f(x)-\frac{1}{2} \frac{f^{\prime}(b)-f^{\prime}(a)}{b-a}(x-b)^{2} .
$$

Escolhendo um função auxiliar adequada obtemos a seguinte variação do Teorema de ÇakmakTiryaki.

Teorema 7 ([4]). Se $\mathrm{f}:[\mathrm{a}, \mathrm{b}] \rightarrow \mathbb{R}$ é uma função derivável em $[\mathrm{a}, \mathrm{b}]$, então, existe $\eta \in(\mathrm{a}, \mathrm{b})$ tal que

$$
\mathrm{f}(\mathrm{b})-\mathrm{f}(\eta)=\mathrm{f}^{\prime}(\eta)(\mathrm{b}-\eta)+\frac{\mathrm{n}-1}{\mathrm{n}} \frac{\mathrm{f}^{\prime}(\mathrm{b})-\mathrm{f}^{\prime}(\mathrm{a})}{(\mathrm{b}-\mathrm{a})^{\mathrm{n}-1}}(\mathrm{~b}-\eta)^{\mathrm{n}}, \quad \mathrm{n} \in \mathbb{N} .
$$

Demonstração. Seja $n \in \mathbb{N}$ e considere a função auxiliar $\phi:[a, b] \rightarrow \mathbb{R}$ dada por

$$
\phi(\mathrm{x})=\mathrm{f}(\mathrm{x})+\lambda(\mathrm{x}-\mathrm{b})^{\mathrm{n}}
$$

onde $\lambda \in \mathbb{R}$. Vamos escolher $\lambda$ tal que a condição $\phi^{\prime}(\mathrm{a})=\phi^{\prime}(\mathrm{b})$ esteja satisfeita.

A função $\phi$ é derivável em $[\mathrm{a}, \mathrm{b}]$ e $\phi^{\prime}(\mathrm{x})=\mathrm{f}^{\prime}(\mathrm{x})+\mathrm{n} \lambda(\mathrm{x}-\mathrm{b})^{\mathrm{n}-1}$. Então,

$$
\begin{aligned}
\phi^{\prime}(\mathrm{a})=\phi^{\prime}(\mathrm{b}) & \Leftrightarrow \mathrm{f}^{\prime}(\mathrm{a})+\mathrm{n} \lambda(\mathrm{a}-\mathrm{b})^{\mathrm{n}-1}=\mathrm{f}^{\prime}(\mathrm{b}) \\
& \Leftrightarrow \lambda=\frac{1}{\mathrm{n}} \frac{\mathrm{f}^{\prime}(\mathrm{b})-\mathrm{f}^{\prime}(\mathrm{a})}{(\mathrm{a}-\mathrm{b})^{\mathrm{n}-1}} .
\end{aligned}
$$

Assim, temos a função auxiliar

$$
\phi(x)=f(x)+\frac{1}{n} \frac{f^{\prime}(b)-f^{\prime}(a)}{(a-b)^{n-1}}(x-b)^{n}
$$

, a qual satisfaz as condições do Teorema Myers (Theorem 3). Então, existe $\eta \in(a, b)$ tal que

$$
\phi(\mathrm{b})-\phi(\eta)=\phi^{\prime}(\eta)(\mathrm{b}-\eta) .
$$

Dessa última igualdade segue (14). 


\section{Funções auxiliares para o Teorema do Valor Médio de Cauchy}

Nesta seção vamos usar algumas funções auxiliares elementares para demonstrar algumas variações do Teorema do Valor Médio de Cauchy.

Teorema 8 (Teorema de Cauchy). Se $\mathrm{f}, \mathrm{g}:[\mathrm{a}, \mathrm{b}] \rightarrow \mathbb{R}$ são funções contínuas em $[\mathrm{a}, \mathrm{b}]$ e deriváveis em $(\mathrm{a}, \mathrm{b})$, então, existe $\eta \in(\mathrm{a}, \mathrm{b})$ tal que

$$
[\mathrm{f}(\mathrm{b})-\mathrm{f}(\mathrm{a})] \mathrm{g}^{\prime}(\eta)=[\mathrm{g}(\mathrm{b})-\mathrm{g}(\mathrm{a})] \mathrm{f}^{\prime}(\eta) .
$$

Se $\mathrm{g}^{\prime}(\mathrm{x}) \neq 0$ para todo $\mathrm{x} \in(\mathrm{a}, \mathrm{b})$, então,

$$
\frac{f(b)-f(a)}{g(b)-g(a)}=\frac{f^{\prime}(\eta)}{g^{\prime}(\eta)} .
$$

Geometricamente, o Teorema de Cauchy diz que existe uma reta tangente à curva $\gamma:[a, b] \rightarrow \mathbb{R}^{2}$ dada por $\gamma(\mathrm{t})=(\mathrm{f}(\mathrm{t}), \mathrm{g}(\mathrm{t}))$, a qual é paralela à reta que passa pelos pontos $\mathrm{A}=(\mathrm{f}(\mathrm{a}), \mathrm{g}(\mathrm{b})) \mathrm{e}$ $\mathrm{B}=(\mathrm{f}(\mathrm{b}), \mathrm{g}(\mathrm{b}))$ da curva $\gamma$.

Se $\mathrm{g}(\mathrm{x})$ = x no Teorema de Cauchy (Teorema 8), então obtemos o Teorema de Lagrange (Teorema 1).

A demonstração do Teorema de Cauchy [12, Teorema 4.14] consiste em aplicar o Teorema de Rolle à função auxiliar $\mathrm{h}:[\mathrm{a}, \mathrm{b}] \rightarrow \mathbb{R}$ dada por

$$
h(x)=f(x)-f(a)-\frac{f(b)-f(a)}{g(b)-g(a)}[g(x)-g(a)]
$$

Como na demonstração do Teorema de Lagrange cabe aqui a seguinte pergunta: Qual é a origem da função auxiliar $\phi$ usada na demonstração do Teorema de Cauchy?

Uma ligeira modificação da função auxiliar (17) é usada em [8, Teorema 5.11] para demonstrar o Teorema de Cauchy:

$$
h(x)=f(x)-\frac{f(b)-f(a)}{g(b)-g(a)} g(x)
$$

M.Poliferno [10] considerou a função auxiliar $\mathrm{h}:[\mathrm{a}, \mathrm{b}] \rightarrow \mathbb{R}$ dada por

$$
h(x)=\frac{f(x)-m g(x)}{\sqrt{m^{2}+1}},
$$

onde $\mathrm{m}=\frac{\mathrm{f}(\mathrm{b})-\mathrm{f}(\mathrm{a})}{\mathrm{g}(\mathrm{b})-\mathrm{g}(\mathrm{a})}$ é a inclinação de reta $\mathrm{L}$ que passa pelos extremos $\mathrm{A}=(\mathrm{f}(\mathrm{a}), \mathrm{g}(\mathrm{a}))$ e $\mathrm{B}=$ $(\mathrm{f}(\mathrm{b}), \mathrm{g}(\mathrm{b}))$ da curva $\gamma(\mathrm{x})=(\mathrm{f}(\mathrm{x}), \mathrm{g}(\mathrm{x}))$. A função h é a distância de ponto da curva $\gamma$ à reta $\mathrm{L}$, então, geometricamente, $\mathrm{h}(\mathrm{a})=0=\mathrm{h}(\mathrm{b})$. Logo, pelo Teorema de Rolle, segue a conclusão do Teorema de Cauchy.

J. Tong [20] considerou a seguinte função auxiliar $\mathrm{h}:[\mathrm{a}, \mathrm{b}] \rightarrow \mathbb{R}$ dada por

$$
h(x)=f(x)-\frac{f(b)-f(a)}{g(b)-g(a)}\left[g(x)-\frac{g(a)+g(b)}{2}\right]
$$


Claramente vemos que $\mathrm{h}(\mathrm{a})=\frac{\mathrm{f}(\mathrm{a})+\mathrm{f}(\mathrm{b})}{2}=\mathrm{h}(\mathrm{b})$, logo, o Teorema de Rolle dá a conclusão do Teorema de Cauchy.

Observação 2. Modificando ligeiramente a função auxiliar h dada em (19) por J. Tong pela função auxiliar

$$
h(x)=f(x)-\frac{f(a)+f(b)}{2}-\frac{f(b)-f(a)}{g(b)-g(a)}\left[g(x)-\frac{g(a)+g(b)}{2}\right]
$$

também obtemos a conclusão do Teorema do Valor Médio de Cauchy.

M. Spiegel [16] considerou a função auxiliar $\mathrm{h}:[\mathrm{a}, \mathrm{b}] \rightarrow \mathbb{R}$ da forma

$$
\mathrm{h}(\mathrm{x})=\mathrm{f}(\mathrm{x})-[\alpha+\beta \mathrm{g}(\mathrm{x})]
$$

onde $\alpha$ e $\beta$ são números reais a serem determinados satisfazendo a condição $\mathrm{h}(\mathrm{a})=\mathrm{h}(\mathrm{b})$. Dessa condição, obtemos $\beta=\frac{\mathrm{f}(\mathrm{b})-\mathrm{f}(\mathrm{a})}{\mathrm{g}(\mathrm{b})-\mathrm{g}(\mathrm{a})}$. Para determinar a constante $\alpha$ fazemos $\mathrm{h}(\mathrm{a})=0$, assim $\alpha=$ $f(a)-\frac{f(b)-f(a)}{g(b)-g(a)} g(a)$. Logo, a função auxiliar tem a forma

$$
h(x)=f(x)-f(a)-\frac{f(b)-f(a)}{g(b)-g(a)}[g(x)-g(a)] .
$$

Note que a função dada em (21) é a mesma função auxiliar considerada por M. Protter e C. Morrey (veja (17)) e, uma forma reduzida da função dada em (21) é

$$
h(x)=f(x)-\frac{f(b)-f(a)}{g(b)-g(a)} g(x),
$$

a qual é obtida quando tomamos $\alpha=0$.

Seguindo a ideia de M. Spiegel em [16], consideramos a função auxiliar $\mathrm{h}:[\mathrm{a}, \mathrm{b}] \rightarrow \mathbb{R}$ dada por

$$
h(x)=f(x)+\lambda g(x),
$$

onde $\lambda \in \mathbb{R}$, o qual será escolhido tal que $h(a)=h(b)$. Dessa condição, obtemos $\lambda=-\frac{f(b)-f(a)}{g(b)-g(a)}$. Assim, temos a função auxiliar

$$
h(x)=f(x)-\frac{f(b)-f(a)}{g(b)-g(a)} g(x) .
$$

Veja que essa função auxiliar é a mesma obtida em (22).

Em 2000, E.Wachnicki ([21, Teorema 3]) demonstrou a seguinte variação do Teorema do Valor Médio de Cauchy.

Teorema 9 (Teorema de Wachnicki [21]). Sejam $\mathrm{f}, \mathrm{g}:[\mathrm{a}, \mathrm{b}] \rightarrow \mathbb{R}$ funções deriváveis em [a, b]. Se $\mathrm{g}^{\prime}(\mathrm{x}) \neq 0$ para todo $\mathrm{x} \in[\mathrm{a}, \mathrm{b}] e$

$$
\frac{f^{\prime}(a)}{g^{\prime}(a)}=\frac{f^{\prime}(b)}{g^{\prime}(b)}
$$


então, existe $\eta \in(\mathrm{a}, \mathrm{b})$ tal que

$$
\frac{\mathrm{f}(\eta)-\mathrm{f}(\mathrm{a})}{\mathrm{g}(\eta)-\mathrm{g}(\mathrm{a})}=\frac{\mathrm{f}^{\prime}(\eta)}{\mathrm{g}^{\prime}(\eta)}
$$

E.Wachnicki em [21, Teorema 4] removeu a condição (23) e obteve o seguinte resultado o qual é um análogo ao Teorema de Sahoo-Riedel.

Teorema 10. Sejam $\mathrm{f}, \mathrm{g}:[\mathrm{a}, \mathrm{b}] \rightarrow \mathbb{R}$ funções deriváveis em $[\mathrm{a}, \mathrm{b}]$. Se $^{\prime}(\mathrm{x}) \neq 0$ para todo $\mathrm{x} \in[\mathrm{a}, \mathrm{b}]$, então, existe $\eta \in(\mathrm{a}, \mathrm{b})$ tal que

$$
\frac{\mathrm{f}(\eta)-\mathrm{f}(\mathrm{a})}{\mathrm{g}(\eta)-\mathrm{g}(\mathrm{a})}=\frac{\mathrm{f}^{\prime}(\eta)}{\mathrm{g}^{\prime}(\eta)}-\frac{1}{2}\left(\frac{\mathrm{f}^{\prime}(\mathrm{b})}{\mathrm{g}^{\prime}(\mathrm{b})}-\frac{\mathrm{f}^{\prime}(\mathrm{a})}{\mathrm{g}^{\prime}(\mathrm{a})}\right) \frac{\mathrm{g}(\eta)-\mathrm{g}(\mathrm{a})}{\mathrm{g}(\mathrm{b})-\mathrm{g}(\mathrm{a})} .
$$

A demonstração desse Teorema (veja [21, Teorema 4]) consiste em aplicar o Teorema de Wachnicki à função auxiliar $\psi:[\mathrm{a}, \mathrm{b}] \rightarrow \mathbb{R}$ dada por

$$
\psi(x)=f(x)-\frac{1}{2}\left(\frac{f^{\prime}(b)}{g^{\prime}(b)}-\frac{f^{\prime}(a)}{g^{\prime}(a)}\right) \frac{(g(x)-g(a))^{2}}{g(b)-g(a)} .
$$

Em [5] mostramos a seguinte variação do Teorema 10.

Teorema 11 ([5, Teorema 2.3]). Sejam $\mathrm{f}, \mathrm{g}:[\mathrm{a}, \mathrm{b}] \rightarrow \mathbb{R}$ funções deriváveis em $[\mathrm{a}, \mathrm{b}]$. Se $\mathrm{g}^{\prime}(\mathrm{x}) \neq 0$ para todo $\mathrm{x} \in[\mathrm{a}, \mathrm{b}]$, então, existe $\eta \in(\mathrm{a}, \mathrm{b})$ tal que

$$
\frac{\mathrm{f}(\eta)-\mathrm{f}(\mathrm{a})}{\mathrm{g}(\eta)-\mathrm{g}(\mathrm{a})}=\frac{\mathrm{f}^{\prime}(\eta)}{\mathrm{g}^{\prime}(\eta)}-\frac{\mathrm{n}-1}{\mathrm{n}}\left(\frac{\mathrm{f}^{\prime}(\mathrm{b})}{\mathrm{g}^{\prime}(\mathrm{b})}-\frac{\mathrm{f}^{\prime}(\mathrm{a})}{\mathrm{g}^{\prime}(\mathrm{a})}\right)\left(\frac{\mathrm{g}(\eta)-\mathrm{g}(\mathrm{a})}{\mathrm{g}(\mathrm{b})-\mathrm{g}(\mathrm{a})}\right)^{\mathrm{n}-1}, \mathrm{n} \in \mathbb{N} .
$$

Demonstração. Seja $n \in \mathbb{N}$ e consideremos a função auxiliar $\psi:[\mathrm{a}, \mathrm{b}] \rightarrow \mathbb{R}$ dada por

$$
\psi(\mathrm{x})=\mathrm{f}(\mathrm{x})+\lambda(\mathrm{g}(\mathrm{x})-\mathrm{g}(\mathrm{a}))^{\mathrm{n}}, \lambda \in \mathbb{R} .
$$

Vamos escolher $\lambda$ de modo que a seguinte condição esteja satisfeita

$$
\frac{\psi^{\prime}(\mathrm{a})}{\mathrm{g}^{\prime}(\mathrm{a})}=\frac{\psi^{\prime}(\mathrm{b})}{\mathrm{g}^{\prime}(\mathrm{b})} \text {. }
$$

A função $\psi$ é derivável em $[\mathrm{a}, \mathrm{b}]$ e $\psi^{\prime}(\mathrm{x})=\mathrm{f}^{\prime}(\mathrm{x})+\lambda \mathrm{n}(\mathrm{g}(\mathrm{x})-\mathrm{g}(\mathrm{a}))^{\mathrm{n}-1} \mathrm{~g}^{\prime}(\mathrm{x})$. De (27) obtemos

$$
\begin{aligned}
\frac{\psi^{\prime}(\mathrm{a})}{\mathrm{g}^{\prime}(\mathrm{a})}=\frac{\psi^{\prime}(\mathrm{b})}{\mathrm{g}^{\prime}(\mathrm{b})} & \Leftrightarrow \frac{\mathrm{f}^{\prime}(\mathrm{a})}{\mathrm{g}^{\prime}(\mathrm{a})}=\frac{\mathrm{f}^{\prime}(\mathrm{b})}{\mathrm{g}^{\prime}(\mathrm{b})}+\lambda \mathrm{n}[\mathrm{g}(\mathrm{b})-\mathrm{g}(\mathrm{a})]^{\mathrm{n}-1} \\
& \Leftrightarrow \lambda=-\frac{1}{\mathrm{n}}\left(\frac{\mathrm{f}^{\prime}(\mathrm{b})}{\mathrm{g}^{\prime}(\mathrm{b})}-\frac{\mathrm{f}^{\prime}(\mathrm{a})}{\mathrm{g}^{\prime}(\mathrm{a})}\right) \frac{1}{(\mathrm{~g}(\mathrm{~b})-\mathrm{g}(\mathrm{a}))^{\mathrm{n}-1}}
\end{aligned}
$$

Assim, a função auxiliar $\psi$ tem a forma

$$
\psi(x)=f(x)-\frac{1}{n}\left(\frac{f^{\prime}(b)}{g^{\prime}(b)}-\frac{f^{\prime}(a)}{g^{\prime}(a)}\right) \frac{(g(x)-g(a))^{n}}{(g(b)-g(a))^{n-1}} .
$$


Agora, aplicando o Teorema de Wachnicki (Teorema 9) às funções $\psi$ e g, existe $\eta \in(a, b)$ tal que

$$
\frac{\psi(\eta)-\psi(\mathrm{a})}{\mathrm{g}(\eta)-\mathrm{g}(\mathrm{a})}=\frac{\psi^{\prime}(\eta)}{\mathrm{g}^{\prime}(\eta)}
$$

De onde segue a igualdade (26)

Observação 3. Se $\mathrm{g}(\mathrm{x})=\mathrm{x}$, então, os Teoremas 9 e 10 transformam-se nos Teoremas de Flett (Teorema 2) e de Sahoo-Riedel (Teorema 4), respectivamente.

Observação 4. Se $\mathrm{g}(\mathrm{x})=\mathrm{x}$, então, o Teorema 11 transform-se em uma variação do Teorema de Sahoo-Riedel (veja [4]), i.e.,

$$
\mathrm{f}(\eta)-\mathrm{f}(\mathrm{a})=\mathrm{f}^{\prime}(\eta)(\eta-\mathrm{a})-\frac{\mathrm{n}-1}{\mathrm{n}} \frac{\mathrm{f}^{\prime}(\mathrm{b})-\mathrm{f}^{\prime}(\mathrm{a})}{(\mathrm{b}-\mathrm{a})^{\mathrm{n}-1}}(\eta-\mathrm{a})^{\mathrm{n}}, \mathrm{n} \in \mathbb{N} .
$$

Em [5], provamos a seguinte variação do Teorema de Wachnicki.

Teorema 12 ([5, Teorema 2.4]). Sejam $\mathrm{f}, \mathrm{g}:[\mathrm{a}, \mathrm{b}] \rightarrow \mathbb{R}$ funções deriváveis em $[\mathrm{a}, \mathrm{b}]$. Se $\mathrm{g}^{\prime}(\mathrm{x}) \neq 0$ para todo $\mathrm{x} \in[\mathrm{a}, \mathrm{b}] e$

$$
\frac{f^{\prime}(a)}{g^{\prime}(a)}=\frac{f^{\prime}(b)}{g^{\prime}(b)}
$$

então, existe $\eta \in(\mathrm{a}, \mathrm{b})$ tal que

$$
\frac{\mathrm{f}(\mathrm{b})-\mathrm{f}(\eta)}{\mathrm{g}(\mathrm{b})-\mathrm{g}(\eta)}=\frac{\mathrm{f}^{\prime}(\eta)}{\mathrm{g}^{\prime}(\eta)}
$$

Removendo a condição (29), obtivemos uma variação do Teorema 12, o qual é um análogo do Teorema Çakmak-Tiryaki (Teorema 6).

Teorema 13. Sejam $\mathrm{f}, \mathrm{g}:[\mathrm{a}, \mathrm{b}] \rightarrow \mathbb{R}$ funções deriváveis em $[\mathrm{a}, \mathrm{b}]$. Se $\mathrm{g}^{\prime}(\mathrm{x}) \neq 0$ para todo $\mathrm{x} \in[\mathrm{a}, \mathrm{b}]$, então, existe $\eta \in(\mathrm{a}, \mathrm{b})$ tal que

$$
\frac{\mathrm{f}(\mathrm{b})-\mathrm{f}(\eta)}{\mathrm{g}(\mathrm{b})-\mathrm{g}(\eta)}=\frac{\mathrm{f}^{\prime}(\eta)}{\mathrm{g}^{\prime}(\eta)}+\frac{1}{2}\left(\frac{\mathrm{f}^{\prime}(\mathrm{b})}{\mathrm{g}^{\prime}(\mathrm{b})}-\frac{\mathrm{f}^{\prime}(\mathrm{a})}{\mathrm{g}^{\prime}(\mathrm{a})}\right) \frac{\mathrm{g}(\mathrm{b})-\mathrm{g}(\eta)}{\mathrm{g}(\mathrm{b})-\mathrm{g}(\mathrm{a})} .
$$

Para demonstrar esse teorema, usamos a função auxiliar $\phi:[a, b] \rightarrow \mathbb{R}$ dada por

$$
\phi(x)=f(x)-\frac{1}{2}\left(\frac{f^{\prime}(b)}{g^{\prime}(b)}-\frac{f^{\prime}(a)}{g^{\prime}(a)}\right) \frac{\left((g(x)-g(b))^{2}\right.}{g(b)-g(a)}
$$

junto com a função g, e logo aplicamos o Teorema 12.

Em [5] obtivemos a seguinte variação do Teorema 13.

Teorema 14 ([5, Teorema 2.5]). Sejam $\mathrm{f}, \mathrm{g}:[\mathrm{a}, \mathrm{b}] \rightarrow \mathbb{R}$ funções deriváveis em $[\mathrm{a}, \mathrm{b}]$. Se $\mathrm{g}^{\prime}(\mathrm{x}) \neq 0$ para todo $\mathrm{x} \in[\mathrm{a}, \mathrm{b}]$, então, existe $\eta \in(\mathrm{a}, \mathrm{b})$ tal que

$$
\frac{\mathrm{f}(\mathrm{b})-\mathrm{f}(\eta)}{\mathrm{g}(\mathrm{b})-\mathrm{g}(\eta)}=\frac{\mathrm{f}^{\prime}(\eta)}{\mathrm{g}^{\prime}(\eta)}+\frac{\mathrm{n}-1}{\mathrm{n}}\left(\frac{\mathrm{f}^{\prime}(\mathrm{b})}{\mathrm{g}^{\prime}(\mathrm{b})}-\frac{\mathrm{f}^{\prime}(\mathrm{a})}{\mathrm{g}^{\prime}(\mathrm{a})}\right)\left(\frac{\mathrm{g}(\mathrm{b})-\mathrm{g}(\eta)}{\mathrm{g}(\mathrm{b})-\mathrm{g}(\mathrm{a})}\right)^{\mathrm{n}-1}, \mathrm{n} \in \mathbb{N} .
$$


Demonstração. Seja $n \in \mathbb{N}$, e consideremos a função auxiliar $\phi:[a, b] \rightarrow \mathbb{R}$ dada por

$$
\phi(\mathrm{x})=\mathrm{f}(\mathrm{x})+\lambda(\mathrm{g}(\mathrm{x})-\mathrm{g}(\mathrm{b}))^{\mathrm{n}}, \lambda \in \mathbb{R} .
$$

Vamos escolher $\lambda$ de modo que a condição seja satisfeita

$$
\frac{\phi^{\prime}(\mathrm{a})}{\mathrm{g}^{\prime}(\mathrm{a})}=\frac{\phi^{\prime}(\mathrm{b})}{\mathrm{g}^{\prime}(\mathrm{b})} \text {. }
$$

A função $\phi$ é derivável em $[\mathrm{a}, \mathrm{b}]$ e $\phi^{\prime}(\mathrm{x})=\mathrm{f}^{\prime}(\mathrm{x})+\lambda \mathrm{n}(\mathrm{g}(\mathrm{x})-\mathrm{g}(\mathrm{b}))^{\mathrm{n}-1} \mathrm{~g}^{\prime}(\mathrm{x})$. Assim,

$$
\begin{aligned}
\frac{\phi^{\prime}(\mathrm{a})}{\mathrm{g}^{\prime}(\mathrm{a})}=\frac{\phi^{\prime}(\mathrm{b})}{\mathrm{g}^{\prime}(\mathrm{b})} & \Leftrightarrow \frac{\mathrm{f}^{\prime}(\mathrm{a})}{\mathrm{g}^{\prime}(\mathrm{a})}+\mathrm{n} \lambda[\mathrm{g}(\mathrm{a})-\mathrm{g}(\mathrm{b})]^{\mathrm{n}-1}=\frac{\mathrm{f}^{\prime}(\mathrm{b})}{\mathrm{g}^{\prime}(\mathrm{b})} \\
& \Leftrightarrow \lambda=\frac{1}{\mathrm{n}}\left(\frac{\mathrm{f}^{\prime}(\mathrm{b})}{\mathrm{g}^{\prime}(\mathrm{b})}-\frac{\mathrm{f}^{\prime}(\mathrm{a})}{\mathrm{g}^{\prime}(\mathrm{a})}\right) \frac{1}{(\mathrm{~g}(\mathrm{a})-\mathrm{g}(\mathrm{b}))^{\mathrm{n}-1}} .
\end{aligned}
$$

Logo, a função auxiliar $\phi$ tem a forma

$$
\phi(x)=f(x)+\frac{1}{n}\left(\frac{f^{\prime}(b)}{g^{\prime}(b)}-\frac{f^{\prime}(a)}{g^{\prime}(a)}\right) \frac{(g(x)-g(b))^{n}}{(g(a)-g(b))^{n-1}} .
$$

Como as funções $\phi$ e g satisfazem as hipóteses do Teorema 12, existe $\eta \in(\mathrm{a}, \mathrm{b})$ tal que

$$
\frac{\phi(\mathrm{b})-\phi(\eta)}{\mathrm{g}(\mathrm{b})-\mathrm{g}(\eta)}=\frac{\phi^{\prime}(\eta)}{\mathrm{g}^{\prime}(\eta)} .
$$

Dessa última igualdade segue o resultado.

\section{Funções auxiliares para o Teorema do Valor Médio Integral}

Nesta seção vamos usar algumas funções auxiliares elementares para demonstrar algumas variações do Teorema do Valor Médio Integral.

Teorema 15 (Teorema do Valor Médio Integral). Se $\mathrm{f}:[\mathrm{a}, \mathrm{b}] \rightarrow \mathbb{R}$ é uma função contínua, então, existe $\xi \in(\mathrm{a}, \mathrm{b})$ tal que

$$
(b-a) f(\xi)=\int_{a}^{b} f(x) d x
$$

Geometricamente, para uma função f não negativa, o Teorema do Valor Médio Integral diz que a área compreendida entre o gráfico de $f$, as retas $x=a, x=b$ e o eixo x é igual a $(b-a) f(\xi)$, ou seja, a área do retângulo de lados b-a e $\mathrm{f}(\xi)$.

Em 1970, S.G. Wayment ([22]) demonstrou a primeira variação do Teorema do Valor Médio Integral, o qual é a versão integral do Teorema de Flett ([2]).

Teorema 16 (Teorema de Wayment). Se $\mathrm{f}:[\mathrm{a}, \mathrm{b}] \rightarrow \mathbb{R}$ é uma função contínua em $[\mathrm{a}, \mathrm{b}]$ e f(a) = f(b), então, existe $\xi \in(\mathrm{a}, \mathrm{b})$ tal que

$$
(\xi-\mathrm{a}) \mathrm{f}(\xi)=\int_{\mathrm{a}}^{\xi} \mathrm{f}(\mathrm{x}) \mathrm{dx}
$$


O seguinte resultado é uma ligeira modificação do Teorema de Wayment.

Teorema 17 ([14, Teorema 3.2]). Se $\mathrm{f}:[\mathrm{a}, \mathrm{b}] \rightarrow \mathbb{R}$ é uma função contínua em $[\mathrm{a}, \mathrm{b}]$ e $\mathrm{f}(\mathrm{a})=\mathrm{f}(\mathrm{b})$, então, existe $\xi \in(\mathrm{a}, \mathrm{b})$ tal que

$$
(b-\xi) f(\xi)=\int_{\xi}^{b} f(x) d x
$$

Em 2007, P.K. Sahoo [14, Teorema 2.1] removeu a condição de fronteira, f(a) = f(b), do Teorema de Wayment (Teorema 16), e obteve o seguinte resultado:

Teorema 18 (Teorema de Sahoo). Se $\mathrm{f}:[\mathrm{a}, \mathrm{b}] \rightarrow \mathbb{R}$ é uma função contínua em $[\mathrm{a}, \mathrm{b}]$, então, existe $\xi \in(\mathrm{a}, \mathrm{b})$ tal que

$$
(\xi-a) f(\xi)-\frac{1}{2} \frac{f(b)-f(a)}{b-a}(\xi-a)^{2}=\int_{a}^{\xi} f(x) d x .
$$

Para demonstrar o Teorema de Sahoo aplicamos o Teorema de Wayment à função auxiliar $\psi$ : $[\mathrm{a}, \mathrm{b}] \rightarrow \mathbb{R}$ dada por

$$
\psi(x)=f(x)-\frac{f(b)-f(a)}{b-a}(x-a)
$$

Em [6] demonstramos a seguinte variante do Teorema de Sahoo.

Teorema 19. Se $\mathrm{f}:[\mathrm{a}, \mathrm{b}] \rightarrow \mathbb{R}$ é uma função contínua em $[\mathrm{a}, \mathrm{b}]$, então, existe $\xi \in(\mathrm{a}, \mathrm{b})$ tal que

$$
(\xi-a) f(\xi)-\frac{n}{n+1} \frac{f(b)-f(a)}{(b-a)^{n}}(\xi-a)^{n+1}=\int_{a}^{\xi} f(x) d x, \quad n \in \mathbb{N} .
$$

Demonstração. Seja $\mathrm{n} \in \mathbb{N}$ e considere a função auxiliar $\psi:[\mathrm{a}, \mathrm{b}] \rightarrow \mathbb{R}$ dada por $\psi(\mathrm{x})=\mathrm{f}(\mathrm{x})+$ $\lambda(\mathrm{x}-\mathrm{a})^{\mathrm{n}}$, onde $\lambda \in \mathbb{R}$. Vamos escolher $\lambda$ de modo que a condição $\psi(\mathrm{a})=\psi(\mathrm{b})$ seja satisfeita. Então,

$$
\mathrm{f}(\mathrm{a})=\mathrm{f}(\mathrm{b})+\lambda(\mathrm{b}-\mathrm{a})^{\mathrm{n}} \Leftrightarrow \lambda=-\frac{\mathrm{f}(\mathrm{b})-\mathrm{f}(\mathrm{a})}{(\mathrm{b}-\mathrm{a})^{\mathrm{n}}} .
$$

Assim, temos a função auxiliar $\psi(\mathrm{x})=\mathrm{f}(\mathrm{x})-\frac{\mathrm{f}(\mathrm{b})-\mathrm{f}(\mathrm{a})}{(\mathrm{b}-\mathrm{a})^{\mathrm{n}}}(\mathrm{x}-\mathrm{a})^{\mathrm{n}}$, a qual é contínua em [a, b]. Aplicando o Teorema de Wayment à função $\psi$, existe $\xi \in(\mathrm{a}, \mathrm{b})$ tal que

$$
\psi(\xi)(\xi-\mathrm{a})=\int_{\mathrm{a}}^{\xi} \psi(\mathrm{x}) \mathrm{dx} .
$$

Dessa igualdade segue (38).

Observação 5. A equação (38) no Teorema 19 dá uma família de Teoremas de valor médio para integrais.

Se consideramos a sequência de números reais $\left\{\mathrm{I}_{\mathrm{n}}\right\}_{\mathrm{n} \in \mathbb{N}}$ onde

$$
I_{n}=(\xi-a) f(\xi)-\frac{n}{n+1} \frac{f(b)-f(a)}{(b-a)^{n}}(\xi-a)^{n+1},
$$


temos

$$
\lim _{n \rightarrow \infty} I_{n}=(\xi-a) f(\xi) .
$$

Note que:

- Se n = 1 em (38), obtemos o Teorema de Sahoo (Teorem 18). Nesse caso, a função auxiliar é da forma

$$
\psi(x)=f(x)-\frac{f(b)-f(a)}{(b-a)}(x-a)
$$

- Se n = 2 em (38), obtemos uma variação de (37)

$$
(\xi-a) f(\xi)-\frac{2}{3} \frac{f(b)-f(a)}{(b-a)^{2}}(\xi-a)^{3}=\int_{a}^{\xi} f(x) d x .
$$

Nesse caso, temos a função auxiliar

$$
\psi(x)=f(x)-\frac{f(b)-f(a)}{(b-a)^{2}}(x-a)^{2} .
$$

Quando removemos a condição de fronteira $f(a)=f(b)$ no Teorema 17, obtemos o seguinte resultado:

Teorema 20. Se $\mathrm{f}:[\mathrm{a}, \mathrm{b}] \rightarrow \mathbb{R}$ é uma função contínua em $[\mathrm{a}, \mathrm{b}]$, então, existe $\xi \in(\mathrm{a}, \mathrm{b})$ tal que

$$
(b-\xi) f(\xi)+\frac{1}{2} \frac{f(b)-f(a)}{b-a}(b-\xi)^{2}=\int_{\xi}^{b} f(x) d x .
$$

Na demonstração desse teorema, aplicamos o Teorema 17 à função auxiliar $\psi$ : [a,b] $\rightarrow \mathbb{R}$ dada por

$$
\psi(x)=f(x)+\frac{f(b)-f(a)}{b-a}(b-x)
$$

Em [6] mostramos a seguinte variação do Teorem 20.

Teorema 21. Se $\mathrm{f}:[\mathrm{a}, \mathrm{b}] \rightarrow \mathbb{R}$ é uma função contínua em $[\mathrm{a}, \mathrm{b}]$, então, existe $\xi \in(\mathrm{a}, \mathrm{b})$ tal que

$$
(b-\xi) f(\xi)+\frac{n}{n+1} \frac{f(b)-f(a)}{(b-a)^{n}}(b-\xi)^{n+1}=\int_{\xi}^{b} f(x) d x, \quad n \in \mathbb{N} .
$$

Demonstração. Seja $\mathrm{n} \in \mathbb{N}$ e considere a função auxiliar $\psi:[\mathrm{a}, \mathrm{b}] \rightarrow \mathbb{R}$ dada por $\psi(\mathrm{x})=\mathrm{f}(\mathrm{x})+$ $\lambda(\mathrm{x}-\mathrm{b})^{\mathrm{n}}$, onde $\lambda \in \mathbb{R}$. Vamos escolher $\lambda$ de modo que a condição $\psi(\mathrm{a})=\psi(\mathrm{b})$ seja satisfeita. Então,

$$
\psi(a)=\psi(b) \Leftrightarrow f(a)+\lambda(a-b)^{n}=f(b) \Leftrightarrow \lambda=\frac{f(b)-f(a)}{(a-b)^{n}} .
$$

Assim, temos a função auxiliar $\psi(\mathrm{x})=\mathrm{f}(\mathrm{x})+\frac{\mathrm{f}(\mathrm{b})-\mathrm{f}(\mathrm{a})}{(\mathrm{b}-\mathrm{a})^{\mathrm{n}}}(\mathrm{x}-\mathrm{b})^{\mathrm{n}}$ a qual é contínua em [a, b]. Aplicando 
o Teorema 17 à função $\psi$, existe $\xi \in(\mathrm{a}, \mathrm{b})$ tal que

$$
(\mathrm{b}-\xi) \psi(\xi)=\int_{\xi}^{\mathrm{b}} \psi(\mathrm{x}) \mathrm{dx},
$$

a qual implica a igualdade (40).

Observação 6. A equação (40) no Teorema 21 dá uma família de teoremas de valor médio para integrais.

Se consideramos a sequência de números reais $\left\{\mathrm{I}_{\mathrm{n}}^{\prime}\right\}_{\mathrm{n} \in \mathbb{N}}$, onde

$$
I_{n}^{\prime}=(b-\xi) f(\xi)+\frac{n}{n+1} \frac{f(b)-f(a)}{(b-a)^{n}}(b-\xi)^{n+1},
$$

temos

$$
\lim _{\mathrm{n} \rightarrow \infty} I_{\mathrm{n}}^{\prime}=(\mathrm{b}-\xi) f(\xi) .
$$

Note que:

- Se n = 1 em (40), obtemos o Teorema 20. Nesse caso, temos a função função auxiliar

$$
\psi(x)=f(x)+\frac{f(b)-f(a)}{(b-a)}(x-b)
$$

- Se n = 2 in (40), obtemos uma variação de (39)

$$
(b-\xi) f(\xi)+\frac{2}{3} \frac{f(b)-f(a)}{(b-a)^{2}}(b-\xi)^{3}=\int_{\xi}^{b} f(x) d x .
$$

Nesse caso, a função auxiliar é da forma

$$
\psi(x)=f(x)+\frac{f(b)-f(a)}{(b-a)^{2}}(x-b)^{2} .
$$

\section{Referências}

[1] Çakmak, D.; Tiryaki, A. Mean value theorem for holomorphic functions. Electron. J. Differential Equations, N. 34, 6pp., 2012.

[2] Flett, T.M. A mean value problem, Math. Gazette. 42, pp.38-39, 1958.

[3] Lang, S., Undergraduate Analysis. Second edition. Undergraduate Texts in Mathematics. Springer-Verlag, New York, 2005.

[4] Lozada-Cruz, G., Some variants of Lagrange's mean value theorem. Acepted for publication, 2020.

[5] Lozada-Cruz, G., Some variants of Cauchy's mean value theorem. Int. J. Math. Ed. Sci. Tech. Accepted for publication, 2019.

[6] Lozada-Cruz, G., Some variants of Integral mean value theorem. Submitted for publication, 2020 . 
[7] Martínez de la Rosa, F., Panorámica de los Teoremas de Valor Medio. Miscelánea Matemática 47, pp.23-38, 2008.

[8] Mercer, Peter R., More Calculus of a single variable. Undergraduate Texts in Mathematics. Springer, New York, 2014.

[9] Myers, R.E. Some elementary results related to the mean value theorem, The Two-Year College Mathematics Journal, Vol. 8, ํo.1, pp.51-53, 1977.

[10] Poliferno, M. J.; A natural auxiliary function for the Mean Value Theorem. Amer. Math. Monthly 69, $\mathrm{N}^{\circ} .1$, pp.45-47, 1962.

[11] Protter, M.H.; Protter, P.E., Calculus with Analytic Geometric. Fourth Edition. Jones and Bartlett Publishers, Inc., 1988.

[12] Protter, M.H.; Morrey Jr., C.B., A First Course in Real Analysis. Second Edition. Undergraduate Texts in Mathematics, Springer-Verlag New York, Inc., 1991.

[13] Sahoo, P.K.; Riedel, T. Mean Value Theorems and Functional Equations, World Scientific, River Edge, NJ, 1998.

[14] Sahoo, P.K., Some results related to the integral mean value theorem, Int. J. Math. Ed. Sci. Tech. 38(6), 818-822, 2007.

[15] Silverman, H., A simple auxiliary function for the mean value theorem. Coll. Math. J. 20, p. 323, 1989.

[16] Spiegel, Murray R., Mean value theorems and Taylor series. Mathematics Magazine 29, pp.263-266, 1956.

[17] Stewart, J., Calculus Early Transcendentals. Sixth Edition, Thomson Brooks/Cole, 2008.

[18] Tong, J., A generalization of the mean value theorem for Integrals, The College Mathematics Journal, Vol. 33, pp.408-409, 2002.

[19] Tong, J., The mean value theorem generalised to involve two parameters. Math. Gazette, vol. 88, N $\mathrm{N}^{\circ}$ 513, pp.538-540, 2004.

[20] Tong, J., A new auxiliary function for the mean value theorem. Journal of the North Carolina Academy of Science Vol. 121, N.4, pp.174-176, 2005.

[21] Wachnicki, E., Une variante du théoréme de Cauchy de la valeur moyenne, Demonstratio Math. 33. 4, pp.737-740, 2000.

[22] Wayment, S.G., An integral mean value theorem, Math. Gazette. 54, 300-301, 1970.

[23] Yates, R. C., The law of the mean. Amer. Math. Monthly. 66, pp.579-580, 1959.

German Lozada-Cruz

Departamento de Matemática, Instituto de Biociências, Letras e Ciências Exatas (IBILCE), Universidade Estadual Paulista (UNESP),

São José do Rio Preto, Brasil. <german.lozada@unesp.br> 
Publicado: 11/08/2020 